\title{
An Experimental Study on the Fluid Forces Induced by Rotor-Stator Interaction in a Centrifugal Pump
}

\author{
Shijie Guo and Hidenobu Okamoto \\ Ebara Research Co. Ltd., Fujisawa-shi, Japan
}

The pressure fluctuations and the radial fluid forces acting on the impeller, the pressures in the volute, as well as the vibration of the shaft in a centrifugal pump were measured simultaneously, and their relationship was investigated. Experiments were done for various diffuser vanes, flow rates, and rotating speeds. It was demonstrated that both the bladepressure fluctuations and the volute static pressures are nonuniform circumferentially (not axisymmetrical) under off-design operating conditions and that the two have a strong relationship. At high flow rates, the blade pressure fluctuations, induced by rotor-stator interactions, are large in areas where the volute static pressure is low. The traveling directions of the rotating pressure waves, the whirling directions of the radial fluid forces, and the most predominant frequency components of both the fluctuations and the forces are discussed, and an equation for predicting them is introduced. It was also noted that large alternating fluid forces are not necessarily associated with large pressure fluctuations. Furthermore, when measuring the radial fluid forces in the rotating frame, other frequency components, in addition to those related to the products of the diffuser vane number and the rotating frequency, may occur due to the circumferential unevenness of the pressure fluctuations on the impeller. These components are predictable.

Keywords centrifugal pump, fluid force, pressure fluctuation, rotorstator interaction, rotordynamics, vibration

Investigation of fluid forces in turbomachinery has been a key research topic for a long time because of the increasing requirements for high reliability, low vibration, and low noise. The research includes a study of the rotor-stator interaction force,

Received 28 March 2002; accepted 28 March 2002.

Address correspondence to Shijie Guo, Ebara Research Co. Ltd., 2-1, Honfujisawa 4-chome, Fujisawa-shi 251, Japan. E-mail: kaku10019@erc.ebara.co.jp

a typical fluid force that can cause not only serious vibration problems in shafts and pipelines but also failure of impellers and bearings. Many experimental studies have been done on the rotor-stator interaction in centrifugal pumps: flows and pressures inside volutes, diffusers, and impellers (Arndt et al. 1989, 1990; Eisele et al. 1997; Ino and Kasai 1985; Kurokawa and Amasaka 1983; Miner et al. 1989; Shintia and Katz 2000; Tsukamoto et al. 1995; Uno et al. 1995), rotordynamic fluid forces acting on impellers (Ohashi et al. 1988; Ohashi and Shoji 1984; Zhang et al. 2000), and vibrations of shafts and impellers (Guo et al. 1999).

Kurokawa and Amasaka (1983) studied the flow in a twodimensional volute and demonstrated that the static pressures and the velocities in the volute were not uniform circumferentially, that is, not axisymmetrical under off-design operating conditions. Miner and colleagues (1989) measured the flow inside a centrifugal impeller by using a laser velocimeter. They demonstrated that the flow was not uniform circumferentially, even at design point, because of the interaction between the impeller and the volute. As for pressure fluctuations, Ino and Kasai (1985) measured the blade pressure fluctuations in a centrifugal pump and found that the fluctuations depended on flow rates and blade/vane angles, and that the fundamental frequency component was $Z g N$ inside the impeller. Arndt and colleagues $(1989,1990)$ measured the pressure fluctuations on the blades of a two-dimensional centrifugal impeller. They reported that the largest blade-pressure fluctuations occurred at the trailing edge, independently of the diffuser vane configuration, but that the magnitudes depended greatly on the vane number and vane angle. Tsukamoto and colleagues (1995) and Uno and colleagues (1995) measured the pressure fluctuations inside the diffuser of a centrifugal pump and reported that the fluctuations on the suction side of the diffuser vane's leading edge were large and that the fundamental frequency component was $\mathrm{ZrN}$. As for rotordynamic forces, Ohashi and colleagues $(1984,1988)$ investigated the interaction destabilizing forces to the rotor by whirling the impeller with a DC motor and measuring the reaction forces for various whirling and rotating ratios. They concluded that the fluid forces of a free impeller basically had a stabilizing effect, but that in the case of a vaned diffuser, the 
interaction forces might be destabilizing for a small ratio of whirling to rotating. Zhang and colleagues (2000) measured the radial fluid forces with strain gauges installed on the shaft and found that unexpected frequency components in addition to $\mathrm{ZgN}$ might occur. A similar phenomenon was also observed in this experiment. In this article, a physical explanation is given and an equation is presented for predicting the frequency components. It is noted here that one of the authors measured the vibration on an impeller that had the same hydraulic details as the one used in this study and found that sidebands occurred in the frequency spectra, especially at high flow rates (Guo et al. 1999). This article demonstrates that sidebands are a consequence of the circumferential unevenness of the pressure fluctuations.

The above mentioned studies have given us a comprehensive understanding of the mechanisms of the pressure fluctuations induced by rotor-stator interactions. However, our understanding of the relationships among the volute pressures, the impeller pressures, and the radial fluid forces as a result of the integration of the impeller pressures is insufficient as yet. In this study, the pressure fluctuations and the radial fluid forces acting on the impeller, as well as the vibration of the shaft in a centrifugal, vaned diffuser pump were measured simultaneously, and their relationship were investigated. The experiment was performed for various diffuser vanes, flow rates, and rotating speeds. Furthermore, the rotating directions of pressure waves, the whirling directions of radial fluid forces, and the most predominant frequency components are discussed, and an equation is introduced for predicting them.

Rotor-stator interaction may be divided into two different mechanisms: potential flow interaction and wake interaction. In this study, because the radial gap between the impeller blade trailing edge and the diffuser vane leading edge was small (about $1.1 \%$ of the outlet diameter of the impeller; Table 1), potential flow interaction was assumed to be predominant.

\section{EXPERIMENT}

Figure 1 shows a conceptual view of the volute casing of the centrifugal pump used in the experiment. The pump was driven by a motor and the rotating speed was changed by an inverter. The experiment was conducted in a recirculating-water test loop with a suction tank, inside which the pressure could be controlled by a vacuum pump and high-pressure air. The flow rate was adjusted with a discharge valve and measured by a Bently flow meter. The centrifugal impeller was a three-dimensional closed design with six blades. The guide vane was fixed to the casing by bolts and therefore was easily removable from the casing. Three kinds of two-dimensional guide vanes (GV11: $Z g=11, \mathrm{GV7}: Z g=7$, and GV5: $Z g=5$ ) were used. Table 1 lists the specifications of the impeller and the guide vanes.

Ten pressure gauges $(\mathrm{C} 1-\mathrm{C} 10)$ were installed on the casing to measure volute pressures as shown in Figure 1, while twelve pressure gauges were installed on the impeller to measure pres-
TABLE 1

Specifications of the Impeller and the Guide Vanes

\begin{tabular}{ll}
\hline Impeller & Closed \\
Number of blades & 6 \\
Outlet diameter $\mathrm{D}_{2} /$ inlet diameter & $328 / 202 \mathrm{~mm}$ \\
Outlet width $\mathrm{b}_{2}$ & $32 \mathrm{~mm}$ \\
$\quad$ Outlet blade angle & 32 degrees \\
Guide vane GV11 & \\
$\quad$ Number of vanes & 11 \\
Inlet diameter/outlet diameter & $331.5 / 430 \mathrm{~mm}$ \\
$\quad$ Inlet vane angle & 18 degrees \\
Guide vane GV7 & \\
$\quad$ Number of vanes & 7 \\
Inlet diameter/outlet diameter & $331.5 / 430 \mathrm{~mm}$ \\
Inlet vane angle & 16.5 degrees \\
Guide vane GV5 & 5 \\
$\quad$ Number of vanes & $331.5 / 430 \mathrm{~mm}$ \\
Inlet diameter/outlet diameter & 10 degrees \\
Inlet vane angle & \\
\hline
\end{tabular}

sure fluctuations, with three on the pressure sides of the blade trailing edges (P1, P3, and P5), three on the suction sides ( 1 1, S3, and S5), and six on the hub (D1, D3, D5, R1, R3, and R5), as shown in Figure 2. The radial fluid forces acting on the impeller were measured by strain gauges installed on the shaft, as shown in Figure 3. Bridge circuits were made so as not to measure the torsional and axial vibrations, but only the bending vibration. Although the bending from the radial fluid forces and that from the fluid moments were included in the output of the strain gauges, it was assumed that the measured bending strains were all from the radial forces acting on the gravity center of the

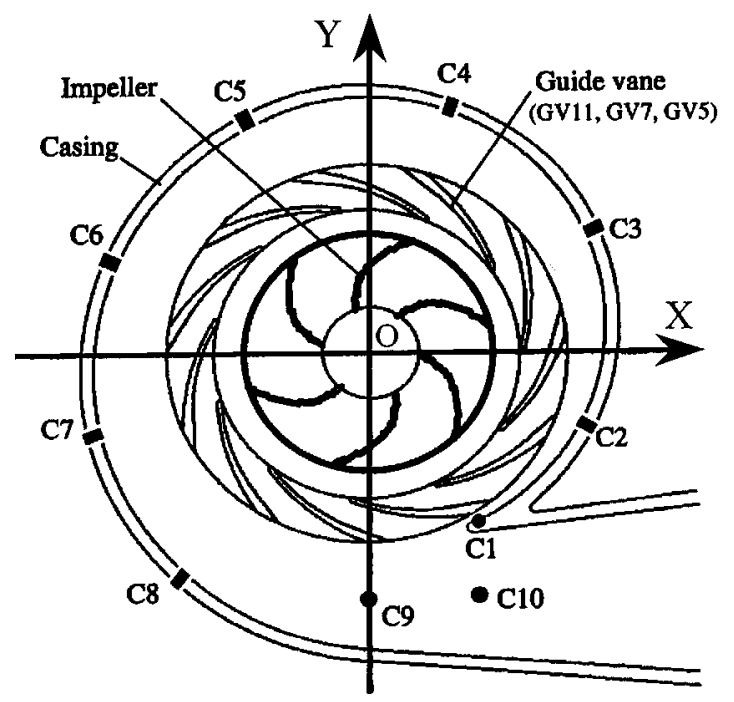

FIGURE 1

Locations of pressure gauges $(\mathrm{C} 1-\mathrm{C} 10)$ on the volute casing and the frame $\mathrm{O}-\mathrm{XY}$, which is fixed to the casing. 


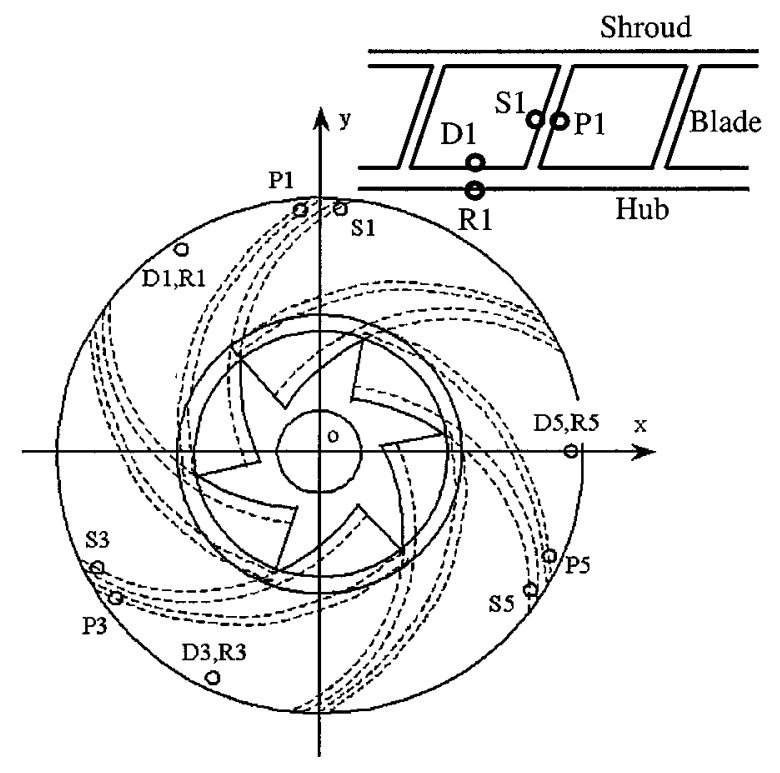

FIGURE 2

Locations of pressure gauges on the impeller and the frame $\mathrm{o}-\mathrm{xy}$, which is fixed to the impeller.

impeller. Meanwhile, the vibration of the shaft was measured by piezoelectric accelerometers installed on the impeller hub (see Fig. 3). All the wires were picked up through the hollow shaft by a slip ring assembly. Because both the radial fluid forces and the vibration of the shaft were measured in the rotating frame, the gravity and buoyancy acting on the impeller influenced the measurement results. This influence was eliminated from the measurement data by using the rotating signals of the shaft as measured by a photoelectric sensor.

The rotating frame was fixed to the impeller, and the relative locations of the coordinate axes in relation to the impeller blades are shown in Figure 2. The fixed frame is fixed to the stationary casing as shown in Figure 1.

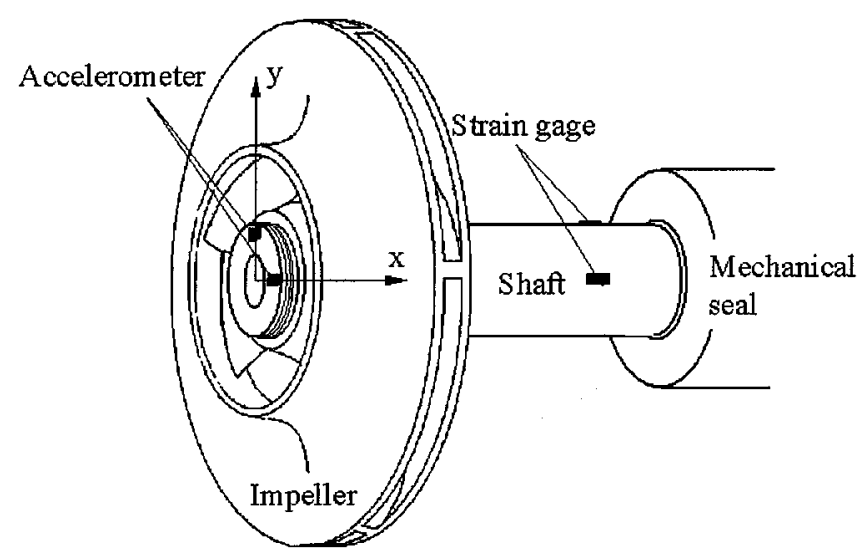

FIGURE 3

Concept view of the impeller and locations of the accelerometers and strain gauges.

\section{RESULTS OF PRESSURE FLUCTUATIONS}

The time history of the pressure fluctuations measured at the pressure side of the impeller blade trailing edge (P1; see Fig. 2) for various flow rates in the case of GV7 is shown in Figure 4. Fluctuations are small near the best efficiency point (BEP) flow rate, and the pressure pulses are upward (pressure increasing) at high flow rates, while downward (pressure decreasing) at partial flow rates. This may be a consequence of the flow patterns around the leading edges of the diffuser vanes. Furthermore, the magnitudes of the pressure pulses are not uniform circumferentially during one revolution of the impeller. This unevenness depends on flow rates. At a high flow rate, the pulses are large when the interaction occurs in the front of the volute exit at C7C9 (see Fig. 1), while the opposite situation can be observed at partial flow rates, that is, the pulses are small when the interaction occurs at $\mathrm{C} 7-\mathrm{C} 9$. It was also confirmed that pressure fluctuations at other measurement points (S1-S5, D1-D5, and $\mathrm{R} 1-\mathrm{R} 5)$ were relatively small compared to those at the pressure sides (P1-P5) of the impeller blades.

Figure 5 shows the pressure fluctuations measured at the impeller blade trailing edge (pressure side, P1) at high flow rates with the GV11 and GV5 guide vanes. Comparisons of Figures 4 and 5 indicate that the circumferential unevenness of the pressure

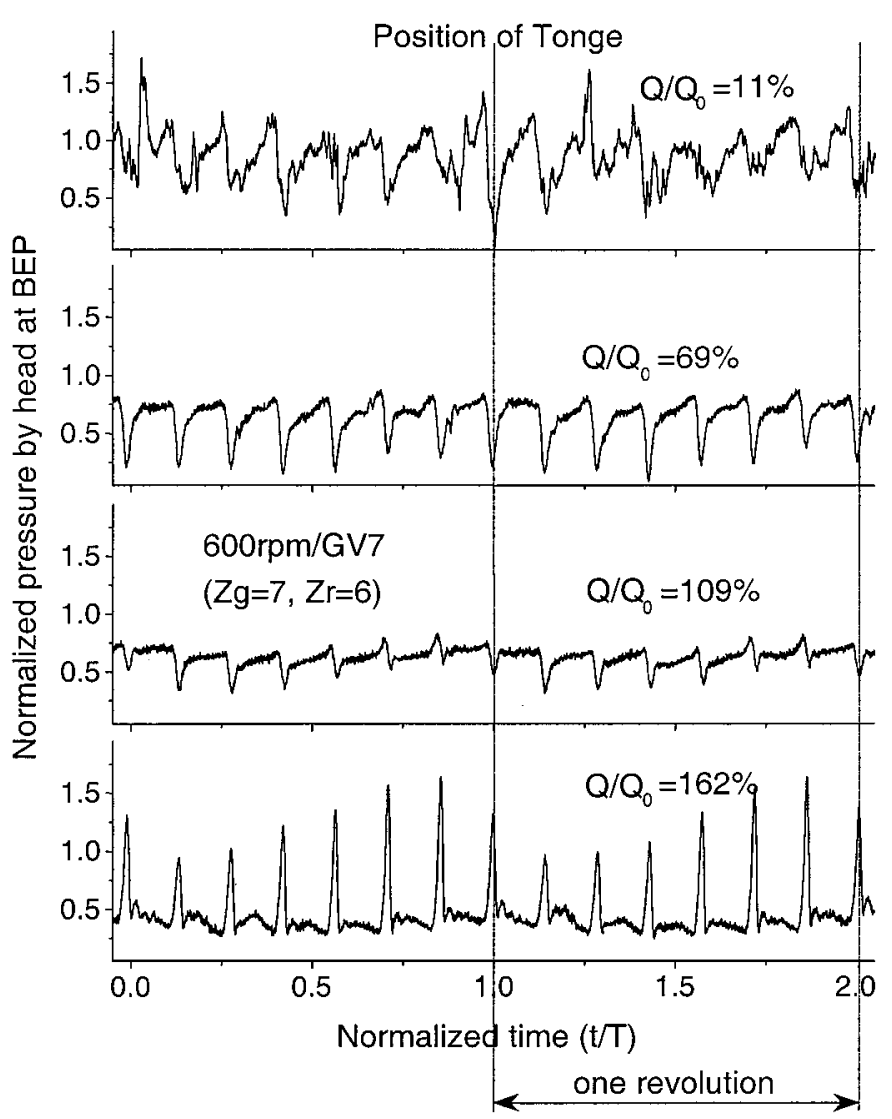

FIGURE 4

Blade pressure fluctuations at P1 in the case of GV7. 


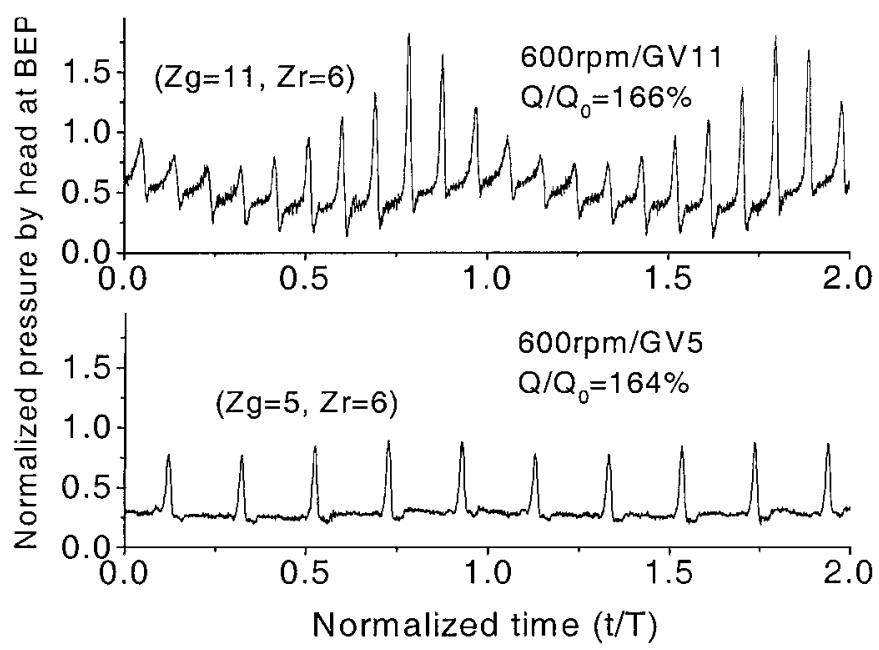

FIGURE 5

Blade pressure fluctuations at P1 with the GV11 and GV5 guide vanes at high flow rates.

fluctuations depends on the number of diffuser vanes. The unevenness is relatively significant and the maximum magnitude of the pulses is large in the case of GV11, while the unevenness is slight and the maximum magnitude is small in the case of GV5. Figure 6 shows the frequency spectra of the blade pressure fluctuations at P1 (see Fig. 2) at high flow rates. In addition to $Z g N$

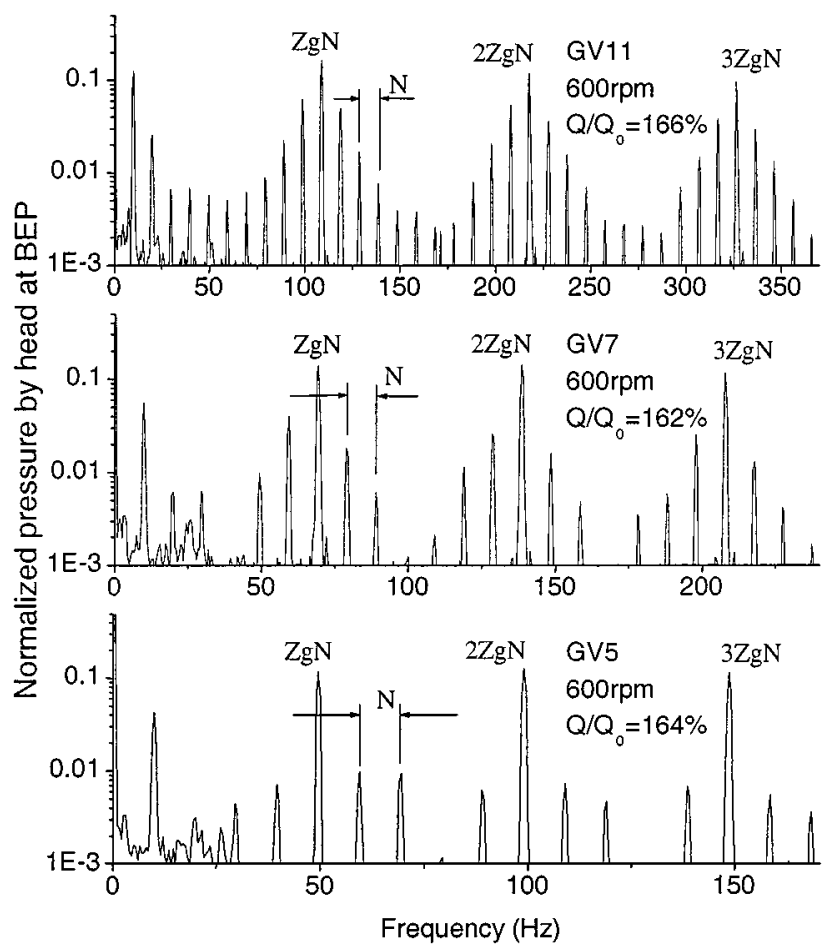

FIGURE 6

Frequency spectra of the blade pressure fluctuations measured at P1, at high flow rates. and its higher harmonic peaks, many side peaks are generated. The frequency difference of two adjacent side peaks is $N$. The side peaks are caused by the circumferential unevenness of the pressure fluctuations. Figure 6 also demonstrates why sidebands were observed in the vibration measurements (Guo et al. 1999).

The circumferential unevenness of the blade pressure fluctuations is strongly related to the distribution of the volute static pressures. Figure 7 shows a comparison between the volute static pressure and the pressure pulses measured at P1 (see Fig. 2), at a high flow rate in the case of GV7. The pressure pulses are large when the interaction occurs in areas where the static pressure is low. This may be attributed to the high flow velocity in areas where the static pressure is low.

Figures 8 and 9 show the frequency spectra of the pressure fluctuations in the volute in cases of GV7 and GV11, measured at $\mathrm{C} 9$ on the casing (see Fig. 1). $\mathrm{ZrN}=6 \mathrm{~N}$ is the most predominant component in cases of GV7 and GV5, while $2 \mathrm{ZrN}=12 \mathrm{~N}$ is the most predominant component in the case of GV11. Furthermore, it was confirmed by the phases of the pressure fluctuations at various measurement points that traveling pressure waves were generated. Figures 10 and 11 show the results, in which the horizontal axes represent circumferential angles, and the vertical axes, the phases based on $\mathrm{P} 1$ and $\mathrm{C} 1$, respectively. Pressure waves were found to travel in the same direction as that of the rotating impeller (forward waves) in cases of GV11 and GV5, in both the rotating and the fixed frames, while they travel in the opposite direction in the case of GV7 (backward waves). This result agrees with that obtained in the measurement of vibration on the impeller (Guo et al. 1999). Traveling directions and the most predominant frequency components of the pressure waves

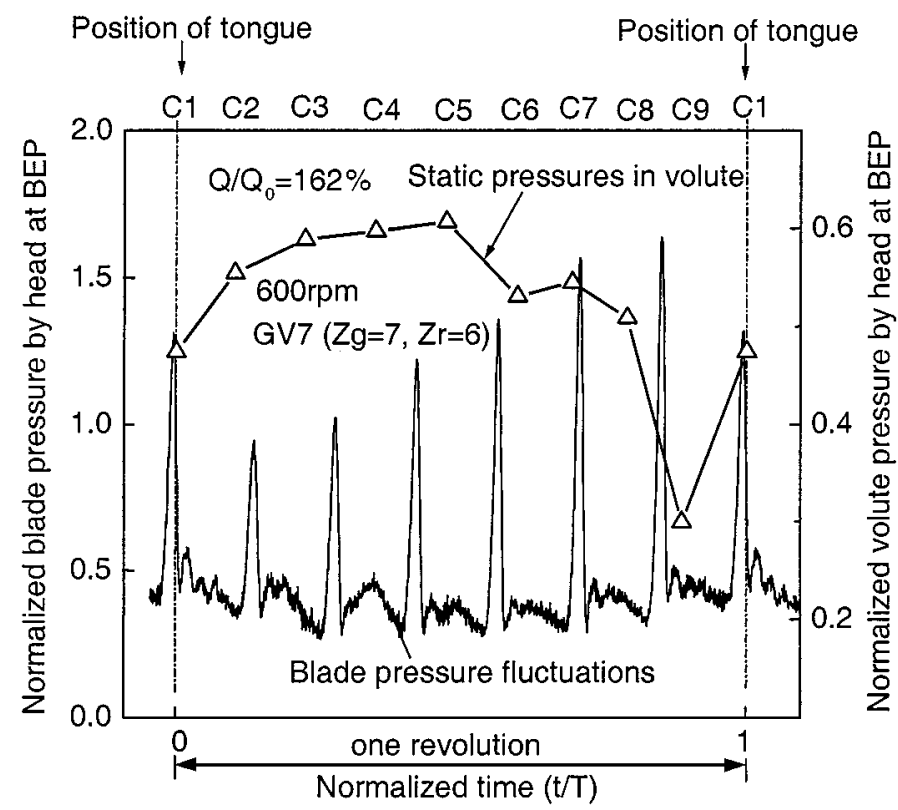

FIGURE 7

Relation between the volute static pressures and the blade pressure fluctuations at $\mathrm{P} 1$. 


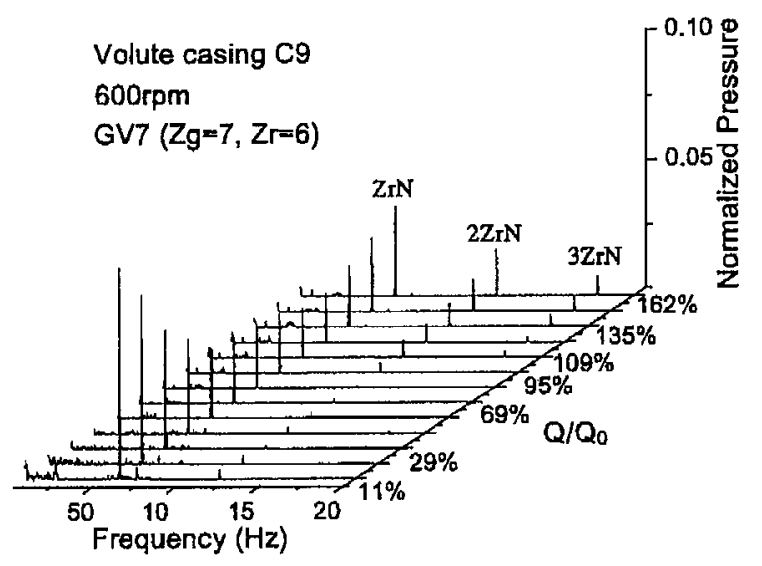

FIGURE 8

Frequency spectra of the pressure fluctuations measured at C9 on the casing in the case of GV7.

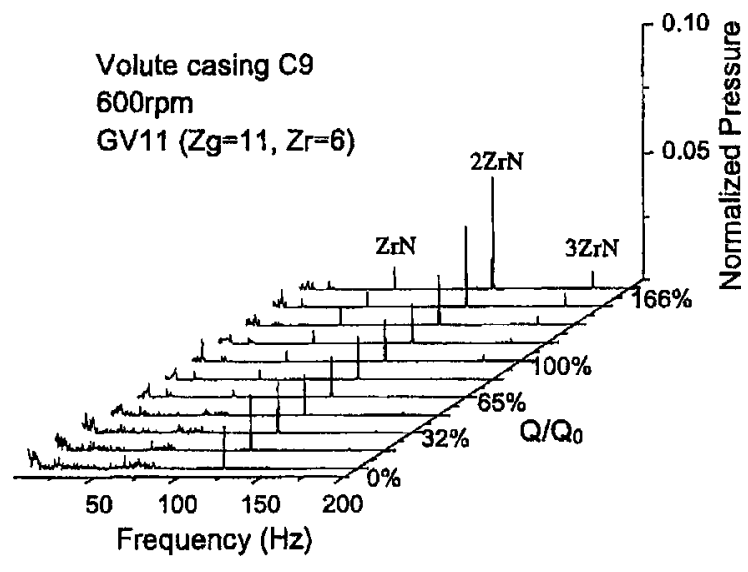

FIGURE 9

Frequency spectra of the pressure fluctuations measured at $\mathrm{C} 9$ on the casing in the case of GV11.

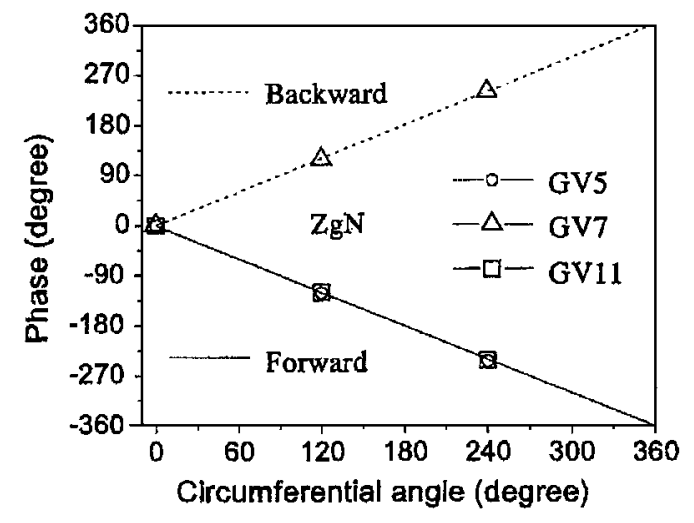

FIGURE 10

Phases of the pressure fluctuations measured at P1-P5 on the blades of the impeller.

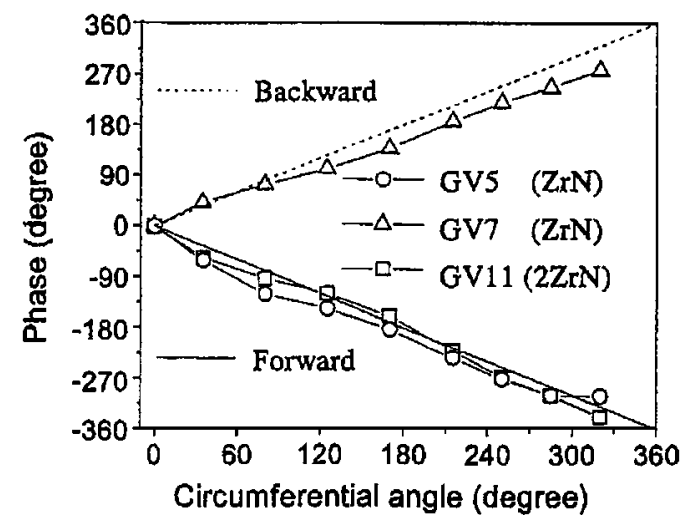

FIGURE 11

Phases of the pressure fluctuations measured at $\mathrm{C} 1-\mathrm{C} 9$ on the casing.

were found not to be strongly dependent on flow rates, but to depend on the combination of the impeller blades and the diffuser vanes. This issue will be discussed in a subsequent section.

\section{RESULTS OF FLUID FORCES}

Frequency spectra of the radial fluid forces in the rotating frame are given in Figure 12 (GV11), Figure 13 (GV7), and Figure 14 (GV5). The forces are shown to be relatively small near the BEP flow rate, while large at high or partial flow rates. As for frequency components, although $\mathrm{ZgN}$ is the most predominant one in all of the three diffuser cases, other components can also be observed: $N$ and $13 N$ in the case of GV11; $N$ and $5 N$ in the case of GV7; and $7 N$ in the case of GV5. Zhang and colleagues, who measured the combination of $(Z g=7, Z r=6)$ and $(Z g=5, Z r=6)$, reported similar results (Zhang et al. 2000).

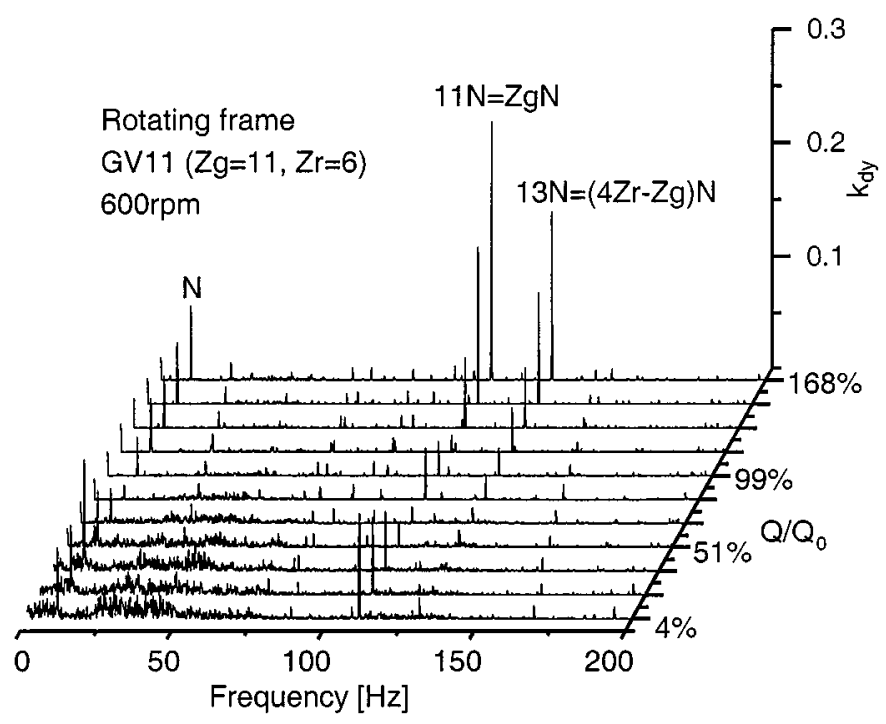

FIGURE 12

Radial fluid forces in the rotating frame (GV11). 


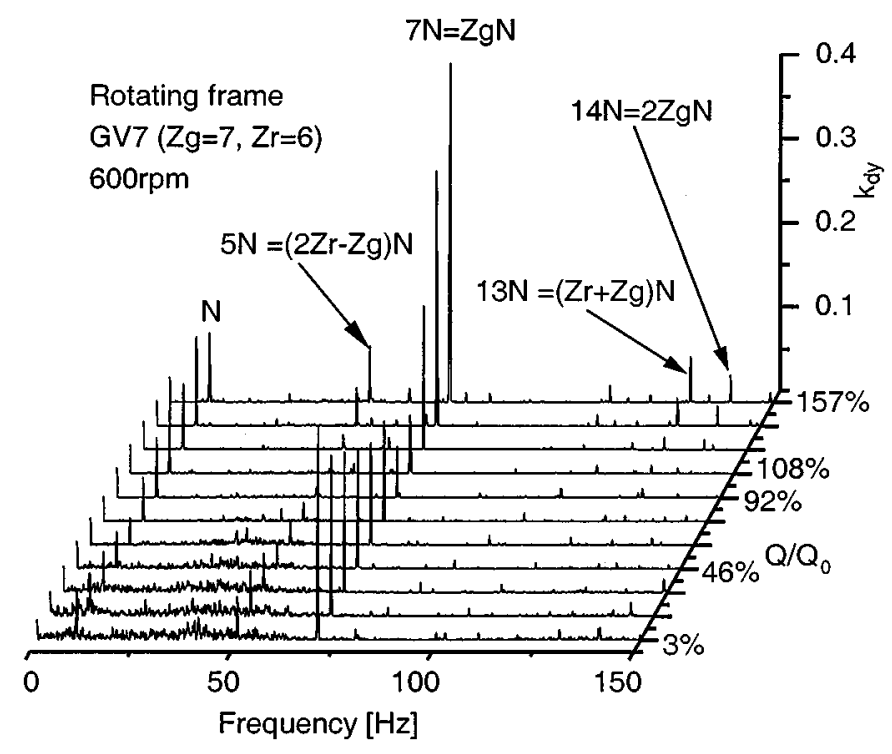

FIGURE 13

Radial fluid forces in the rotating frame (GV7).

We believe that these components occurred due to the circumferential unevenness of the pressure fluctuations (see Figs. 4 and 5). The reason will be discussed in the next section. It is also noted that although the radial fluid forces are an integration of the impeller pressure fluctuations, comparisons between Figures 4 and 5 and Figures 12, 13, and 14 indicate that large pressure fluctuations are not necessarily associated with large fluid forces. The maximum magnitude of the pressure pulses is small in the case of GV5, but the fluid forces are much larger than those in cases of GV11 and GV7.

Lissajous patterns of various frequency components of the radial fluid forces in the rotating frame are shown in Figure 15.

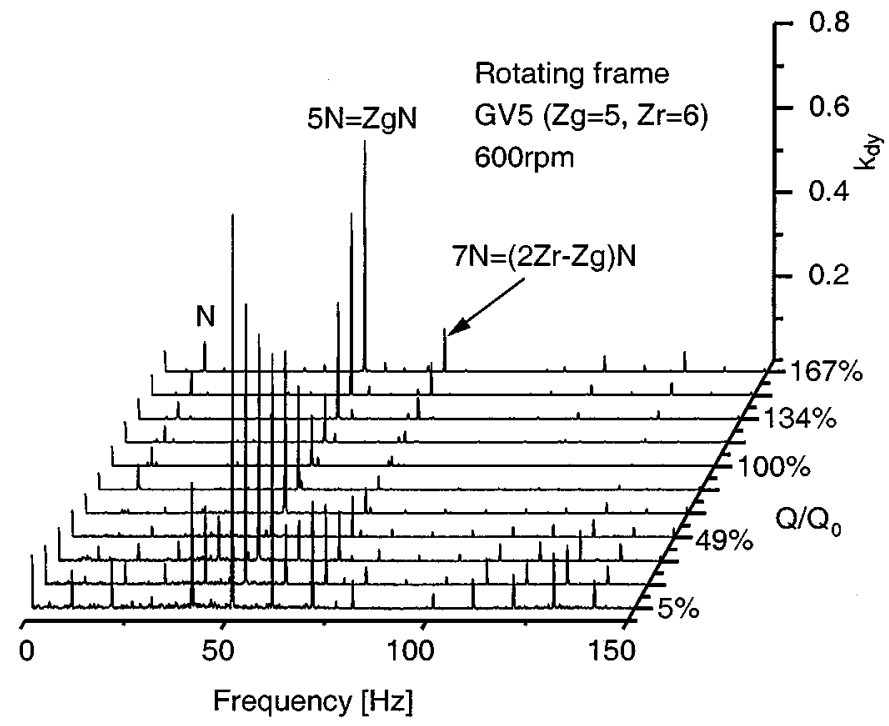

FIGURE 14

Radial fluid forces in the rotating frame (GV5).

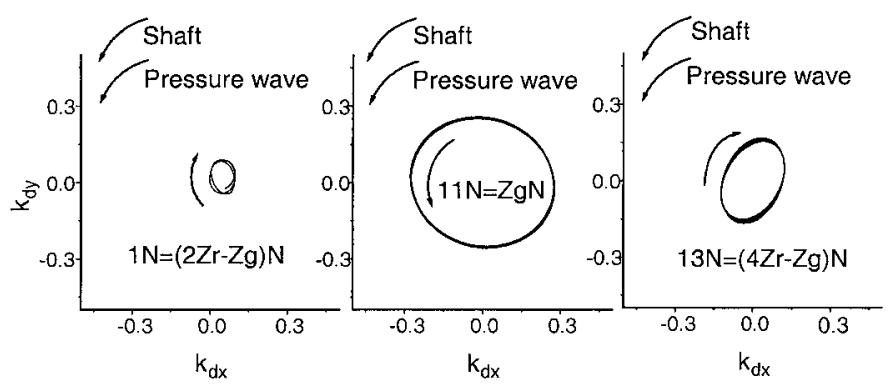

(a) GV11( $\left.\mathrm{Zg}=11,600 \mathrm{rpm}, \mathrm{Q} / \mathrm{Q}_{0}=168 \%\right)$

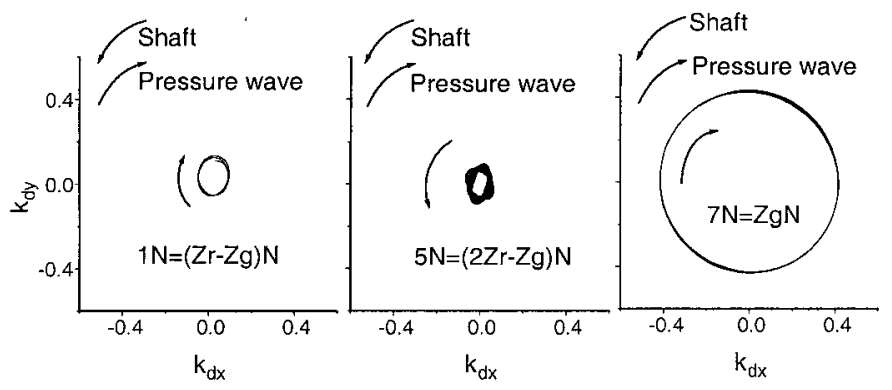

(b) GV7 ( $\left.\mathrm{Zg}=7,600 \mathrm{rpm}, \mathrm{Q} / \mathrm{Q}_{0}=157 \%\right)$

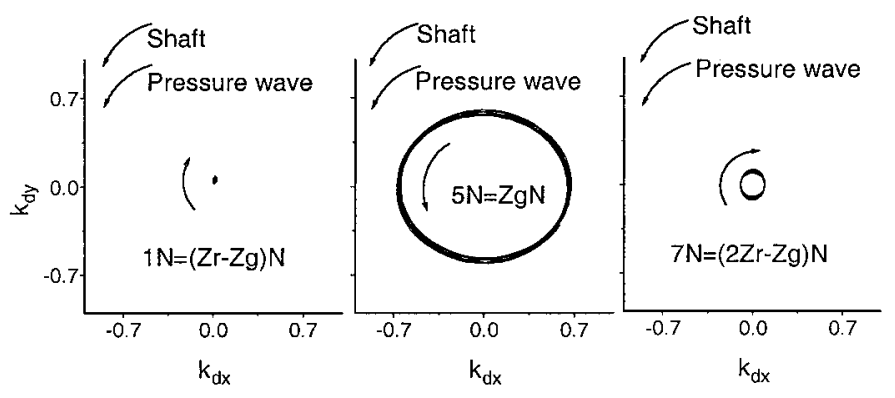

(c) GV5(Zg=5, 600rpm, Q/Q $\left.\mathrm{Q}_{0}=167 \%\right)$

\section{FIGURE 15}

Lissajous patterns of the radial fluid forces in the rotating frame.

The $N$ component rotates in a direction opposite from that of the rotating impeller. This is because the casing rotates in the opposite direction when it is viewed from the rotating frame. The $Z g N$ components rotate in the same direction as the rotating pressure waves. The rotating directions of other components ( $13 N$ in the case of GV11, $5 N$ in the case of GV7, and $7 N$ in the case of GV5) are opposite from those of the $Z g N$ components.

The radial fluid forces in the rotating frame were transformed into the fixed frame, and the frequency spectra thus obtained are shown in Figure 16 (GV11), Figure 17 (GV7), and Figure 18 (GV5), from which we find that $2 Z r N=12 N$ is the most predominant component in the case of GV11, while $Z r N=6 N$ is the most predominant component in the cases of GV7 and GV5. Frequency components will be discussed in the next section. The Lissajous patterns of the fluid forces in the fixed frame are shown 


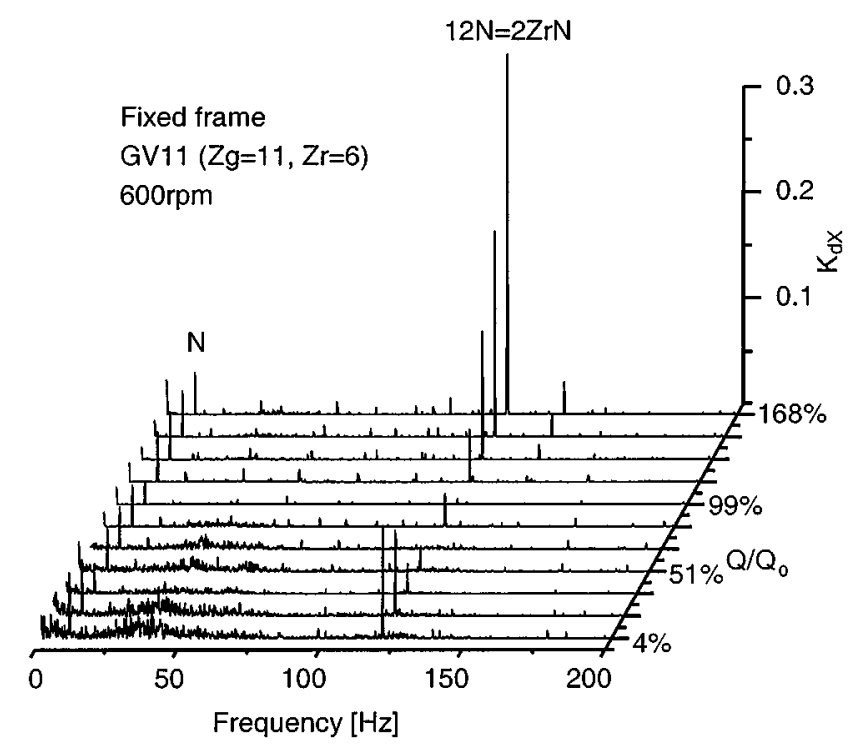

FIGURE 16

Radial fluid forces in the fixed frame (GV11).

in Figure 19. The fluid forces rotate in the same directions as those of the pressure waves, even in the fixed frame. They give a backward excitation to the shaft in the case of GV7, but a forward excitation in the cases of GV11 and GV5. Measurement results on the vibration of the shaft also showed that the shaft whirled backward in the case of GV7 but forward in the cases of GV11 and GV5.

\section{DISCUSSIONS OF FREQUENCY COMPONENTS AND THE ROTATING DIRECTIONS OF PRESSURE WAVES}

The rotating directions of the pressure waves, the whirling directions of the fluid forces as well as their frequency components

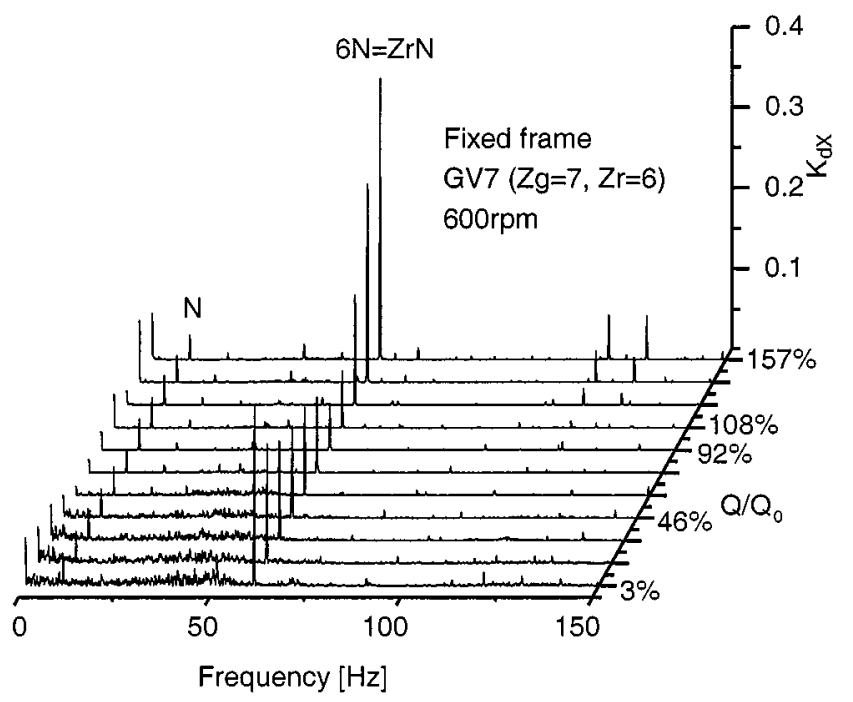

FIGURE 17

Radial fluid forces in the fixed frame (GV7).

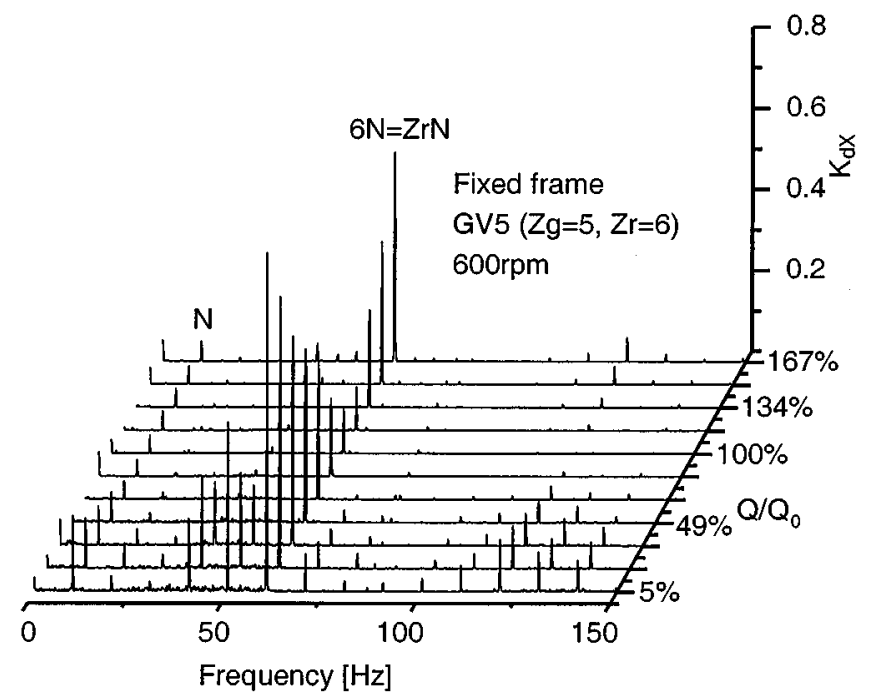

FIGURE 18

Radial fluid forces in the fixed frame (GV5).

will be discussed herein. Figure 20 is a schematic of the interaction between the impeller and the diffuser. If there is no common measure between the blade number and the vane number, interaction occurs once the impeller rotates at an angle of $2 \pi /(\mathrm{ZrZg})$ or, in other words, once the time passes $\Delta t=2 \pi /(N Z r Z g)$. We numbered the impeller blades and the diffuser vanes 0,1 , $2, \ldots$ and assumed that blade 0 was passing through vane 0 at $t=0$, and at $t=\Delta t=2 \pi /(N Z r Z g)$, blade $m$ and vane $n$ will be interacting; then

$$
n \frac{2 \pi}{Z g}-m \frac{2 \pi}{Z r}= \pm \frac{2 \pi}{Z g Z r}
$$

from which we get

$$
n Z r-m Z g= \pm 1 \text {. }
$$

In the fixed frame, the pressure waves rotate at an angle of $2 \pi / Z g$ once the impeller rotates $2 \pi /(Z r Z g)$. The rotating speed of the pressure waves is $n Z r$ times that of the impeller, so $n Z r N$

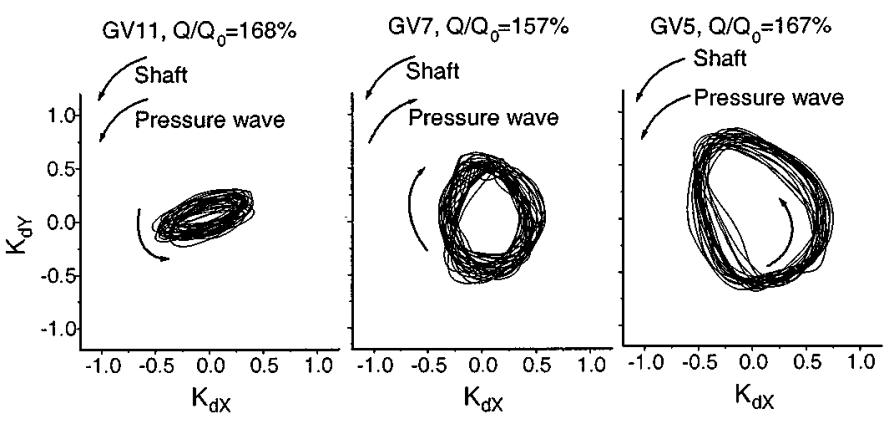

FIGURE 19

Lissajous patterns of the radial fluid forces in the fixed frame at high flow rates. 


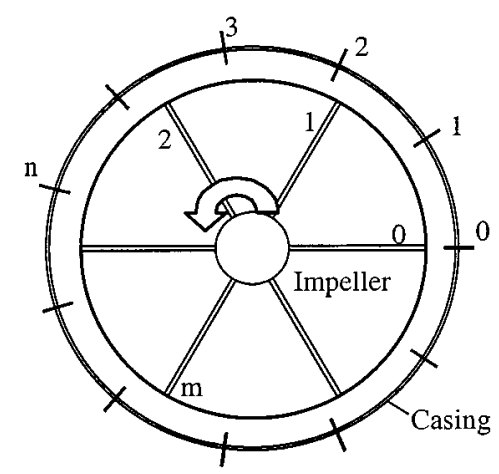

FIGURE 20

Schematic of rotor-stator interaction.

will be the most predominant component. Accordingly, we can conclude that $m Z g N$ is the most predominant component in the rotating frame. Equation (2) demonstrates why $2 Z r N$ is the most predominant component in the fixed frame in the case of GV11 (see Fig. 9, pressure fluctuations; Fig. 16, radial fluid forces), while $Z r N$ is the most predominant component in the cases of GV7 and GV5. Furthermore, the pressure waves rotate in the same direction as the impeller in the case of the plus sign in Equation (2), while they rotate in the opposite direction in the case of the minus sign, regardless of whether the pressure waves are measured in the rotating or the fixed frames. Table 2 gives a list of the traveling directions of the rotating pressure waves estimated by Equation (2). The estimation agrees with the measured results. Equation (2) has been found valid only in areas where traveling pressure waves exist. Inside the impeller or the diffuser, where no rotating pressure waves are generated, the fundamental frequency may be the blade/vane passing frequency and the most predominant component will be $Z r N$ (inside the diffuser) and $Z g N$ (inside the impeller) (Ino and Kasai 1985; Tsukamoto et al. 1995; Uno et al. 1995).

Let us here consider the frequency components of the radial fluid forces measured in the rotating frame. Suppose that at time $t=\Delta t=2 \pi /(N Z r Z g)$, blade $m$ and vane $n$ are interacting (see Fig. 20), and a force $\mathbf{P}_{m, n}$ is generated. Then at time $t=h \Delta t=$ $2 \pi h /(N Z r Z g)$, blade $h m$ and vane $h n$ will be interacting, and a force $\mathbf{P}_{h m, h n}$ will be generated. Furthermore, we suppose, for convenience, that the force acts toward the center of the impeller (the direction of $\mathbf{P}_{h m, h n}$ is not important in the discussion of frequency components). Then the force in the $x$ direction of the

TABLE 2

$m, n$ Satisfying $n Z r-m Z g= \pm 1$ and the Traveling Directions of the Pressure Waves Estimated by the Equation

\begin{tabular}{lrrrcl}
\hline$Z r$ & $Z g$ & $m$ & $n$ & Sign & Direction of wave \\
\hline 6 & 11 & 1 & 2 & + & Forward \\
6 & 7 & 1 & 1 & - & Backward \\
6 & 5 & 1 & 1 & + & Forward \\
\hline
\end{tabular}

rotating frame can be expressed as

$$
f_{x}(t)=\mathbf{P}_{h m, h n} \cos (m Z g N t) .
$$

If the magnitude of $\mathbf{P}_{h m, h n}$ is constant- that is, the pressure fluctuations acting on the impeller are uniform circumferentially - the most predominant component of the fluid forces in the rotating frame will be $m Z g N$, where $m$ is an integer satisfying Equation (2). However, the fluctuations are not uniform, and $\mathbf{P}_{h m, h n}$ is not a constant, as shown in Figures 4 and 5. It was confirmed in the experiment that $\mathbf{P}_{h m, h n}$ depends only on $h n$, the position in the volute, rather than on $\mathrm{hm}$. Because $\mathbf{P}_{h m, h n}$ is a periodic function of time, and supposing that it is an even function, then we can express it by a Fourier series as

$$
\begin{aligned}
\mathbf{P}_{h m, h n} & =a_{0}+a_{1} \cos (h n \cdot 2 \pi / Z g)+a_{2} \cos (2 h n \cdot 2 \pi / Z g)+\cdots \\
& =a_{0}+a_{1} \cos (n Z r N t)+a_{2} \cos (2 n Z r N t)+\cdots
\end{aligned}
$$

where $a_{0}, a_{1}, a_{2}, \ldots$ are constants. Disregarding items higher than the second one and substituting Equation (4) into Equation (3), we get

$$
\begin{aligned}
f_{x}(t)= & a_{0} \cos (m Z g N t)+a_{1} \cos (n Z r N t) \cdot \cos (m Z g N t) \\
& +a_{2} \cos (2 n Z r N t) \cdot \cos (m Z g N t) .
\end{aligned}
$$

The frequency component of the first item in Equation (5) is $m Z g N$; the components of the second item are $(n Z r+m Z g) N$ and $(n Z r-m Z g) N=1 N$; those of the third item are $(2 n Z r$ $+m Z g) N$ and $(2 n Z r-m Z g) N$. These are what we observed in the experiment. Table 3 is a list of the frequency components estimated by Equation (5). The estimation agrees with the mea-

\begin{tabular}{|c|c|c|c|c|}
\hline $\mathrm{Zr}$ & $Z g$ & $m$ & $n$ & Frequency Component \\
\hline 6 & 11 & 1 & 2 & $\begin{array}{l}(n Z r-m Z g) \mathrm{N}=(2 Z r-Z g) N=1 N \\
\boldsymbol{m Z g N}=Z \mathbf{g} N=\mathbf{1 1} N \\
(2 n Z r-m Z g) N=(4 Z r-Z g) N=13 N \\
(n Z r+m Z g) N=(2 Z r+Z g) N=23 N \\
(2 n Z r+m Z g) N=(4 Z r+Z g) N=35 N\end{array}$ \\
\hline 6 & 7 & 1 & 1 & $\begin{array}{l}(n Z r-m Z g) N=(Z r-Z g) N=1 N \\
(2 n Z r-m Z g) N=(2 Z r-Z g) N=5 N \\
\boldsymbol{m Z g N}=\mathbf{Z g N}=\mathbf{7 N} \\
(n Z r+m Z g) N=(Z r+Z g) N=13 N \\
(2 n Z r+m Z g) N=(2 Z r+Z g) N=19 N\end{array}$ \\
\hline 6 & 5 & 1 & 1 & $\begin{array}{l}(n Z r-m Z g) N=(Z r-Z g) N=1 N \\
\boldsymbol{m Z g N}=\mathbf{Z g N}=\mathbf{5} N \\
(2 n Z r-m Z g) N=(2 Z r-Z g) N=7 N \\
(n Z r+m Z g) N=(Z r+Z g) N=11 N \\
(2 n Z r+m Z g) N=(2 Z r+Z g) N=17 N\end{array}$ \\
\hline
\end{tabular}
surement shown in Figures 12, 13, and 14.

\section{TABLE 3}

$m, n$ Satisfying $n Z r-m Z g= \pm 1$ and Predominant Frequency Components of Fluid Forces in the Rotating Frame 
Because the radial fluid forces are generated from the rotating pressure waves, the whirling direction of the fluid forces will be the same as the rotating direction of the pressure waves. When the rotating direction of the pressure waves is the same as that of the impeller, the fluid forces will be a forward-whirling excitation to the shaft; otherwise, they will be a backward-whirling excitation. This provides a physical explanation for the fact that the fluid forces are a backward-whirling excitation and the shaft whirls backward in the case of GV7, as shown in Figures 15 and 19.

\section{CONCLUSIONS}

The pressure fluctuations and the radial fluid forces acting on the impeller, the pressures in the volute, as well as the vibration of the shaft in a centrifugal vaned diffuser pump were measured simultaneously, and their relationships were investigated. The experiment was done for various diffuser vanes, flow rates, and rotating speeds. It was found that:

1. Pressure fluctuations are large on the pressure sides of the blade trailing edges of the impeller, as compared with those on the suction sides and the hub. The interaction-induced pressure pulses, which are large at high and partial flow rates, are upward (pressure increasing) at high flow rates, but are downward (pressure decreasing) at partial flow rates. Blade pressure fluctuations at very small flow rates are much more random in time than those at high flow rates.

2. Both the blade pressure fluctuations and the volute static pressures are not uniform circumferentially under off-design operating conditions, and the two have a strong relation with each other. At high flow rates, blade pressure fluctuations are large in areas where the volute static pressure is low. The circumferential unevenness of the fluctuations, which may cause sidebands in frequency spectra, is dependent on flow rates.

3. The rotating directions and the predominant frequency components of both the pressure waves and the radial fluid forces can be described by $n Z r-m Z g= \pm 1$. In the case of the plus sign, the rotating direction of the pressure waves is forward and the radial fluid forces are a forward excitation to the shaft in both the rotating and the fixed frames, whereas the rotating direction of the pressure waves is backward and the radial fluid forces are a backward excitation in the case of the minus sign. The $m Z g N$ component is the most predominant one in the rotating frame, and $n Z r N$ is the most predominant one in the fixed frame. Furthermore, when measuring the radial fluid forces in the rotating frame, other frequency components, such as $(n Z r+m Z g) N,(n Z r-m Z g) N=1 N$, $(2 n Z r-m Z g) N$, and $(2 n Z r+m Z g) N$ may be observed in addition to $m Z g N$ because of the circumferential unevenness of pressure fluctuations acting on the impeller.

4. Although the radial fluid forces are an integration of the pressures acting on the impeller, large alternating fluid forces are not necessarily associated with large pressure fluctuations.

\section{NOMENCLATURE}

$\mathrm{Zr} \quad$ number of blades of the impeller

$Z g \quad$ number of vanes of the diffuser

$m, n, h \quad$ integers

$N \quad$ rotating angular frequency of the impeller $(\mathrm{rad} / \mathrm{sec})$

$K_{d X}, K_{d Y}$ radial fluid forces with respect to the fixed frame (fixed to the casing), normalized by $D_{2} b_{2} H_{0}$

$k_{d x}, k_{d y} \quad$ radial fluid forces with respect to the rotating frame (fixed to the impeller), normalized by $D_{2} b_{2} H_{0}$

$\mathbf{P}_{m, n} \quad$ radial force acting on the impeller when blade $m$ of the impeller and vane $n$ of the diffuser interact

$D_{2} \quad$ outlet diameter of the impeller (m)

$b_{2} \quad$ outlet width of the impeller (m)

$H_{0} \quad$ total head at BEP $(\mathrm{Pa})$

$Q \quad$ flow rate $\left(\mathrm{m}^{3} / \mathrm{s}\right)$

$Q_{0} \quad$ flow rate at $\mathrm{BEP}\left(\mathrm{m}^{3} / \mathrm{s}\right)$

$t, T \quad$ time, rotating period of the impeller, $T=2 \pi / N$ (sec)

BEP best efficiency point

\section{REFERENCES}

Arndt, N., Acosta, A. J., Brennen, C. E., and Caughey, T. K. 1989. Rotor-stator interaction in a diffuser pump. ASME Journal of Turbomachinery 111:213-221.

Arndt, N., Acosta, A. J., Brennen, C. E., and Caughey, T. K. 1990. Experimental investigation of rotor-stator interaction in a centrifugal pump with several vaned diffusers. ASME Journal of Turbomachinery 112:98-108.

Eisele, K., Zhang, Z., Casey, M., Gulich, J., and Schachenmann, A. 1997. Flow analysis in a pump diffuser. 1. LDA and PTV measurements of the unsteady flow. ASME Journal of Fluids Engineering 119:968-977.

Guo, S., Ando, Y., and Tano, M. 1999. An experimental study on vibration of the impeller of a centrifugal pump, 8-15. Transactions of JSME, Paper C, 65-629. In Japanese.

Ino, T., and Kasai, K. 1985. An analysis of unsteady flow induced by interaction of a centrifugal impeller and a vaned diffuser, 154-159. Transactions of JSME, Paper B, 51-471. In Japanese.

Kurokawa, J., and Amasaka, Y. 1983. A study on the flow in a volute casing. 2. Experiment on a two-dimensional logarithmically spiral volute, 2735-2745. Transactions of JSME, Paper B, 49-448. In Japanese.

Miner, S. M., Beaudoin, R. J., and Flack, R. D. 1989. Laser velocimeter measurements in a centrifugal flow pump. ASME Journal of Turbomachinery 111:205-212.

Ohashi, H., Sakurai, A., and Nishihama, J. 1988. Influence of impeller and difusser geometries on the lateral fluid forces of whirling centrifugal impeller, 285-306. NASA Conference Publication 3026.

Ohashi, H., and Shoji, H. 1984. Lateral fluid forces acting on a whirling centrifugal impeller in vaneless and vaned diffuser, 109-122. NASA Conference Publication 2338.

Sinha, M., and Katz, J. 2000. Quantitative visualization of the flow in a centrifugal pump with diffuser vanes I. On flow structures and turbulence. ASME Journal of Fluids Engineering 122:97107. 
Tsukamoto, H., Uno, M., Nagai, Y., Fukuno, H., Hamafuku, N., and Okamura, T. 1995. Pressure fluctuation downstream of diffuser pump impeller, 183-190. Transactions of JSME, Paper B, 61-586. In Japanese.

Uno, M., Tsukamoto, H., Nagai, Y., Fukuno, H., Hamafuku, N., and Okamura, T. 1995. Measurement of unsteady pressure due to rotor-stator interaction in pump guide vane pas- sage, 191-198. Transactions of JSME, Paper B, 61-586. In Japanese.

Zhang, M., Tsukamoto, H., Miyazaki, K., Hashimoto, T., and Qin, W. 2000. Experimental and numerical study of unsteady fluid force due to rotor-stator interaction in a diffuser pump, 48-53. Proceedings of the 6th Asian International Conference on Fluid Machinery. Kuala Lumpur, Malaysia. 

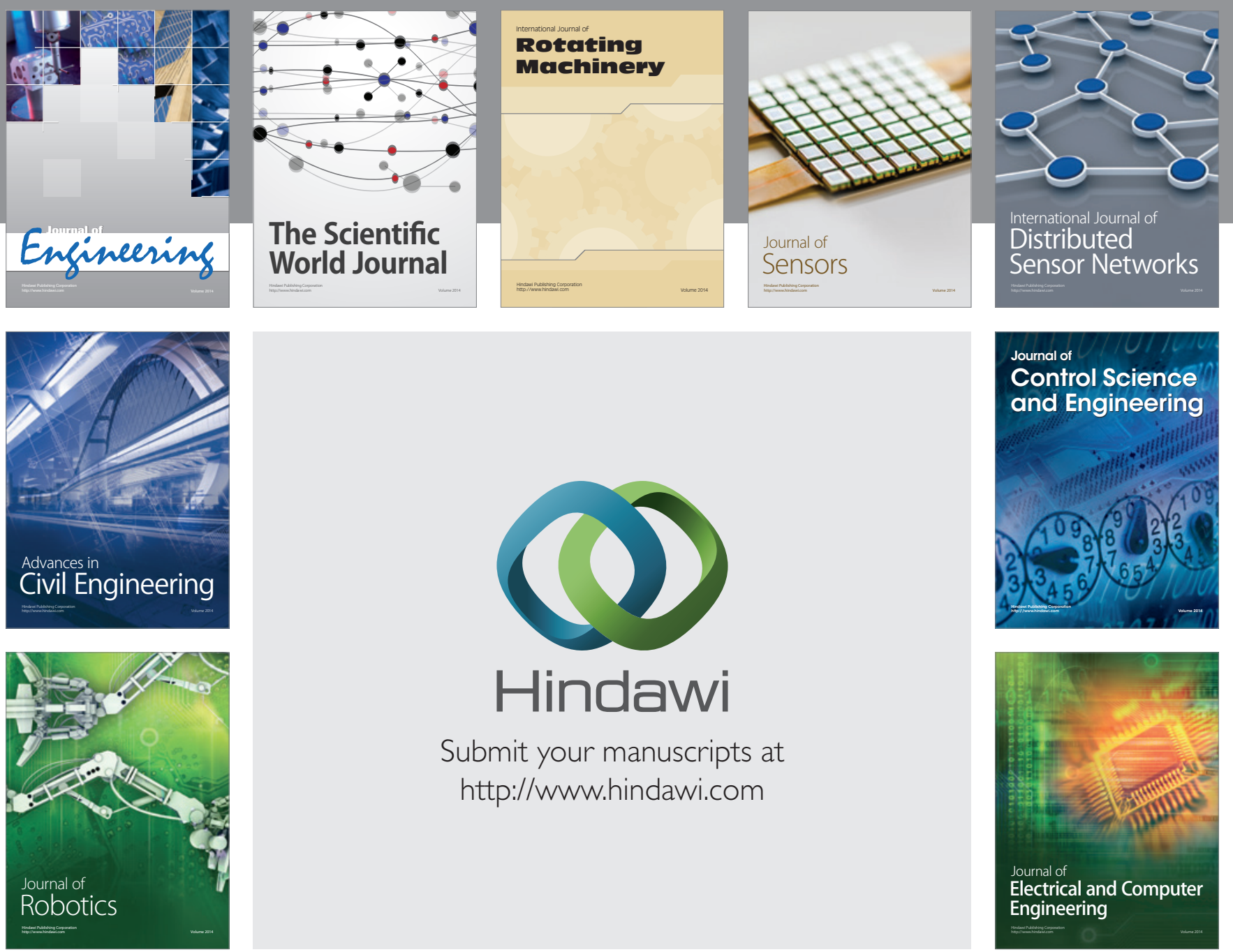

Submit your manuscripts at

http://www.hindawi.com
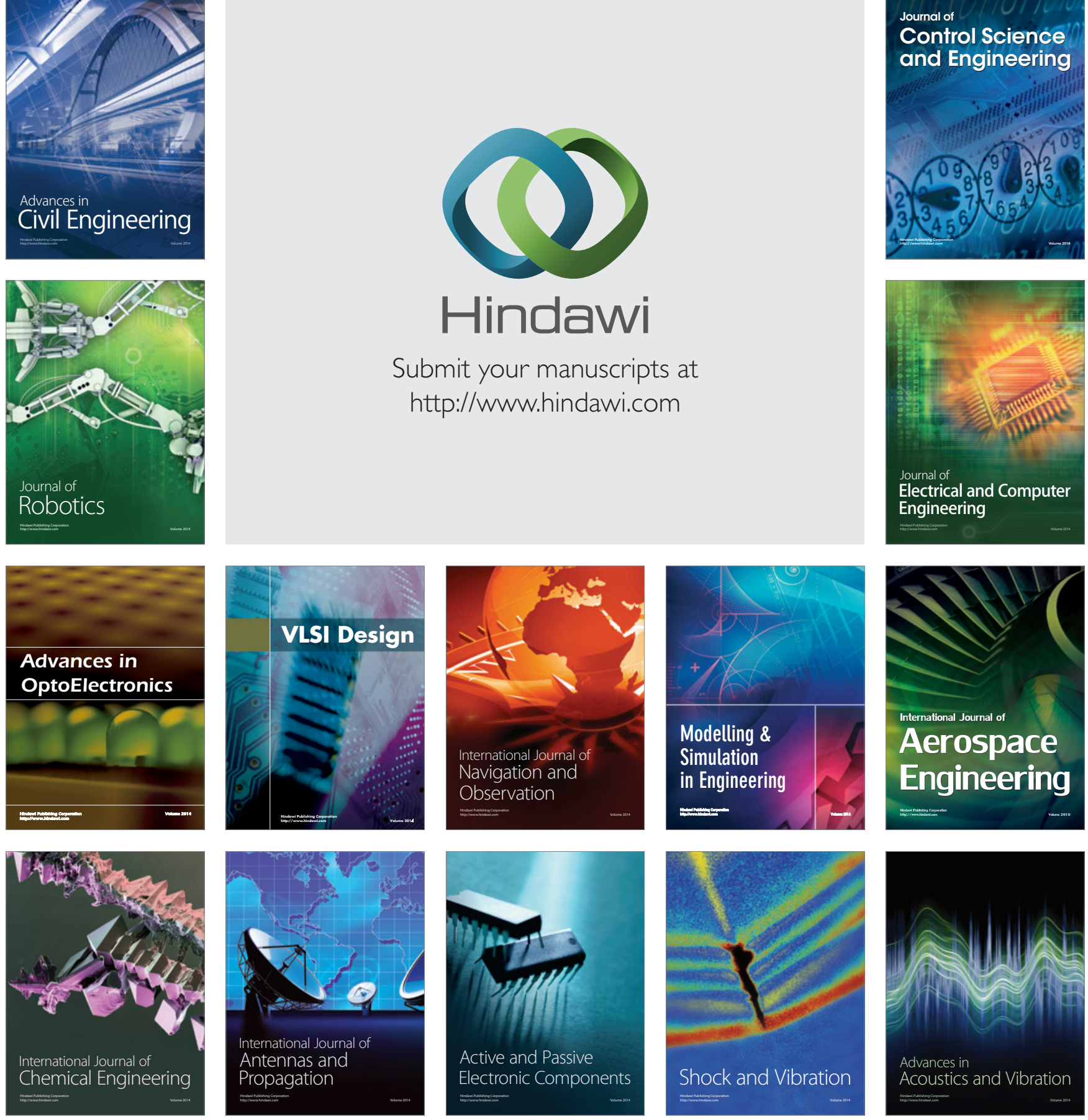